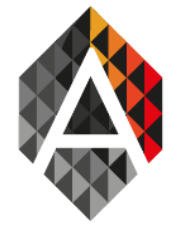

Fábio Silva and Cesar Analide

Algoritmi Centre, University of Minho

fabiosilva@di.uminho.pt, analide@di.uminho.pt

\title{
Tracking Context-Aware Well-Being through Intelligent Environments
}

KEYWORD

\author{
ABSTRACT
}

Well-Being; Intelligent Systems; Sensor Networks

\begin{abstract}
The growth of personal sensors and the ability to sensorize attributes connected with the physical beings and environments are increasing. Initiatives such as Internet of Things (IoT)) aim to connect devices and people through communication channels in order to automate and fuel interaction. Targeted approaches can be found on the Smart Cities pro-jects which use the IoT to gather data from people and attributes related to city management. Though good for management of new cities, well-being should as well be of principal importance. It regards users higher than infrastructure and managerial data. Taking lessons from ergonomic studies, health studies and user habits it is possible to track and monitor user daily living. Moreover, the link between user living conditions and sparse events such as illness, indispositions can be tracked to well-being data through autonomous services. Such application is detailed in the approach categorized by this article and the research presented.
\end{abstract}

\section{Introduction}

The internet of things is a new paradigm, in which every device is digitally connected, regardless of their function and communicates with other devices and other people over communication protocols. The use of sensors and other methods for the acquisition of data and relevant information is common for most projects. Therefore, using data obtained from sensors it is possible to gather data and to develop intelligent systems that have impact on user behaviour. In the subject of intelligent cities, systems with purpose of offering services that are aiming to reduce unnecessary expenses and improving management of available resources are proposed (Longo, Roscia, \& Lazaroiu, 2014).

It applies both to fixed devices and personal devices that accompany people (Atzori, Iera, \& Morabito, 2010). More examples can be enumerated by devices that are being incorporated inside the actual body, such as identification chips, smart tattoos and alike (Steele \& Clarke, 2013). Smart city is a term applied to digital research using computational methods and systems that results in better, easier and faster management of services and goods inside inhabited areas. In this setup, the internet of things acts as a base service which enables smart cities applications to collect information directly from the environment and people and the integrated fusion of data and information. This benefits the planning of actions to improve the status quo. Among other concerns, health, comfort and well-being are topics being addressed in smart cities research (Solanas et al., 2014).

If the technologies described under the concepts of smart cites and internet of things are perceived as social services, then it is possible to gain access to a new set of valuable information on both the environ- 
ment and users. These trends, despite having ethical challenges of their own, present a number of opportunities for society. Sensorization, monitoring and sharing of information are terms intimately connected to the new intelligent systems being created. Even more, applied research related to health organizations and also the well-being of populations or individuals is present in recent studies. Connected environments and the act of monitoring comfort parameters are not under active research but also regulated by governments. For examples, air quality is an area that is actually regulated by governments which define acceptable parameters. Additionally, we could present the project executed in the city of Santander (Krčo, Fernandes, \& Sanchez, 2013), Spain. Besides being profitable, it allows people to access the data that are being captured, such as the location of public transports, traffic control, illumination and water control. In these projects, a person can interact with these systems either by being presented with useful information gathered or challenged based on motivational strategies to engage user into certain behaviours or actions.

Concurrently, research conducted in the field of smart environments studies the impact of air composition in health, concentration tasks and psychological comfort. As expected, research directions are more specialized than government regulations and are being pushed forward by the quality of sensors and sensor networks which portrait better and better images of air composition across time and space inside environments. There are other examples with equal strategies that aim to better assess and diagnose unoptimised or harmful situations with focus on well-being and general health (Piro, Cianci, Grieco, Boggia, \& Camarda, 2014).

The quest for physical well-being is addressed under smart cities by the use of indicators from personal devices such as smartwatches, sport monitors or smart bands which among other things can monitor some health parameters such as heart rate, blood oxygen levels and sport activities. Research in the body response to environment parameters is also potentiated by these personal devices that act as personal sensors (Chan, Estève, Fourniols, Escriba, \& Campo, 2012). Their use to calculate more intrusive parameter such heat balance index for each individual helps perceive whether it is cold or warm in different situations. The environment condition alone is correlated to well-being and comfort status (Rana, Kusy, Jurdak, Wall, \& Hu, 2013). More importantly, there are decisions that can be made using such information that may affect both physical and psychological health. Using fixed sensors over specialized areas are of interest to assess environments and their impact on heath (Atallah, Lo, \& Yang, 2012). For instance, projects that monitor city or indoor air quality provide rich information about potential health risks that may impair both physical and psychological well-being. What is more, the availability of digital services allows a faster identification and actuation upon these threats. The difficulty gathering and constantly monitor such parameters leads to situations where interventions are planned later than they should and the problems worse. Social sustainable indicators developed to assess development of countries and populations can also be automated using sensory networks with devices directly connected with each other that can provide information that would normally require expansive survey, and field tests.

\section{Related Work}

According to the Oxford Dictionary of English, well-being is the state of being comfortable, healthy, or happy. In order to assess it, the three subjects should be covered by the process conducting such evaluation.

In a professional environment, according the World Health Organization a healthy job is likely to be one where the pressures on employees are appropriate in relation to their abilities and resources, to the amount 
of control they have over their work, and to the support they receive from people who matter to them. As health is not merely the absence of disease or infirmity but a positive state of complete physical, mental and social well-being, a healthy working environment is one in which there is not only an absence of harmful conditions.

Environment and physical working conditions are important organizational risk factors (Biron, Ivers, Brun, \& Cooper, 2006). Previous approaches to this work focused on the physical attributes predicament such as heart rate, and comfort due to environment conditions. The assumption that comfort equilibrium calculation was too complex to perform on a recurrent analysis (Fanger, 1970) are mostly proved wrong in an intelligent environment equipped with smart sensors. Although, some of the algorithms might still be considered complex, most mathematical can be used fairly easy by computational systems. From this perspective it is possible to perform scoring systems to rate the degree of comfort a user experiences. Traditional studies use ergonomic research to estimate ideal parameters that are adequate to the space, the people and the task performed. Considering these variables, in this study, we can analyse traditional attributes such as temperature, humidity, luminosity, relevant attributes like gases and airborne dust. However, it is possible to increase the precision of these models, not only estimating individual assessments but also correlated assessments through dedicated indicators with sensor and data fusion techniques. As an example there are a number of indexes that can be created, namely, perfected temperature, percentage of people in discomfort and stress related with heat perception (K. Parsons, 2010) and adequate luminosity for each task. These sensors monitor the environment and its attributes looking for suboptimal attributes, however relate these attributes with user discomfort or user stress is still a relative study. There is another layer, physical human sensors which, in this case, monitor the heart rate for each individual inside smart environments. Whenever suboptimal values are detected, it will be possible to relate the physical state of each person to the environment attributes. Psychological and physiological adverse ambient conditions can produce significantly changes in a person. However, authors orient this topic to a set of variables. In this particular approach sound, temperature and luminosity are studied as external factors that affect well-being and mood states. Previous authors have debated the influence of such variables in the impact of mood change in people (K. C. Parsons, 2000). Related studies between stress and well-being and stress recognition can also be found. In (Choi, Ahmed, \& Gutierrez-Osuna, 2010) we can see a wearable system for ambulatory stress monitoring recording a number of physiological variables known to be influenced by stress. In (Tauseef, 2012) author gathers values from a skin temperature sensor, a heart rate sensor and a skin conductance sensor. The signals from the sensors are input into a microcontroller where all the processing takes place and carried out though ZigBee technology. Data are stored in a computer it is stored for data analysis and feature extraction for emotion recognition.

Comfort is subjective directly related to a person's personality, beliefs and habits. There are however ergonomic studies that provide the necessary background to create an environment which satisfies the most common needs to keep both the people and environments healthy. Although not being an extensive review of possible attributes it does indicate a minimum set of possible attributes to start comfort and well-being assessments (F Silva, Olivares, Royo, Vergara, \& Analide, 2013).

\section{Context Aware Well-Being System Architecture}

A combination of personal and environmental attributes provides a better representation of the information required to assess well-being and hypotheses about user condition, its relation to environment at- 
tributes and the activities being performed. Such information though not being medically considered as hard prove to diagnosis may be used as soft information about condition and habits of living. This system depends on the number of sensing devices and attributes considered to make decisions more accurate and expressive. It is also important to adapt the notions of comfort and safety according to the context of tasks and activities being performed. Taking knowledge from a knowledge base about each environment, for instance relations between sensor and event records, it is actually possible to estimate the state of wellbeing, both currently and in the future.

\subsection{Indicator Design}

Ambient sensorization can be carried out by projects that implement a sensor networks. With such middleware and access to sensor that monitor environmental and behavioural attributes is possible to determine levels of comfort for various attributes such as the temperature or noise. Thermal comfort is typically assessed through complex indicators with several data inputs. In this case there are several indicators in the literature, the current prototype uses the Predicted Mean Value (PMV) index. The PMV index is the average vote estimated from a set of individuals in the environment, and the method which determines this indicator has been developed by Fanger (Charles, 2003)(Fanger, 1970). With the PMV value, using the scale [-3;3] it is possible to find the level of thermal comfort using the specified indicator. An increasing negative score mean more cold discomfort while an increasing positive score mean hot discomfort. The goal is to score around the value zero. This choice for the first prototype was based on familiarity with this indicator but other alternative indicators in the literature exist, such as the Physiological Equivalent Temperature (PET) (Höppe, 1999). A similar characterise is that these indicators take information such as humidity, weather and clothing before their assessment.

According to main area of interest, indicator count tends to become more skewed near the category closely related to institutional or political interests. Indicator design may be implemented using different philosophical strategies as documented in the literature. As an initial analysis it can be measured from bottomup or top-down approaches depending on how the problem is tackled initially. From that point on, the relative perception of the important factors towards comfort play its role. Across the literature, it can be found different definitions for different approaches to monitor and develop such indicator. It is of course of great importance to clearly understand the strategy employed has it has significant importance when translating these indicators into computational systems. As an example if the focus is in studying environments, indicators should be oriented from a top-down approach in which the detail of the indicator portraits our level of detail for such environment. On the other hand, if our focus is geared towards elements of environments such as its users, then a bottom up approach would be best as it would provide detailed information for the element at study which may be generalised to an environment but with lack of generality. 


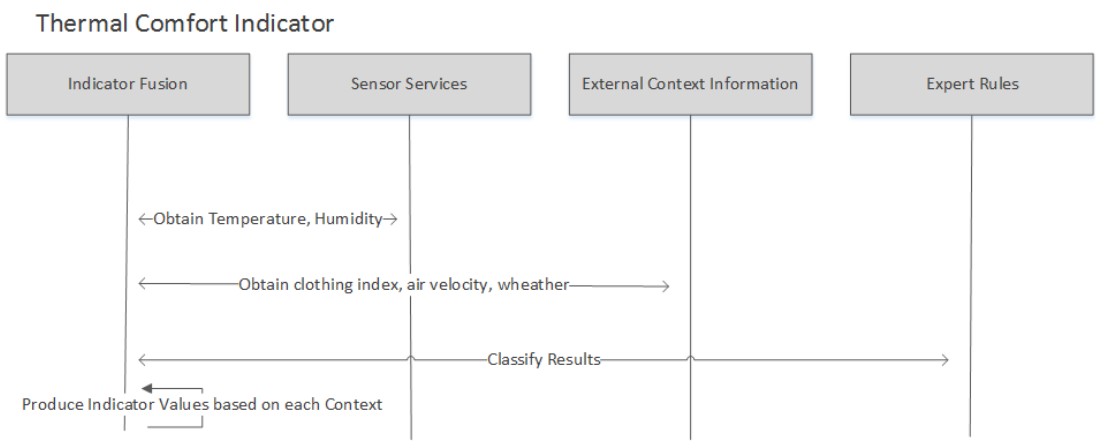

Figure 1 - Process for Indicator Construction

The process of implementing indicator in a computational system often requires indicator fusion. This is done for a number of possible reasons such the amount of data required by the indicator such as the PMV, to combine reading from the same type of sensors or to merge sensor data to information available in other communication channels. In its most complete form, indicator fusion is done according to the flow depicted in Figure 1 the thermal comfort indicator. Other examples may use sensor data directly skipping most portions of this workflow of only use a subset of them. This is dependent on the indicator implementation in the design process.

\subsection{Environment Evaluation}

A home environment is a particular setup, with a very personal context. Depending on job state, a person may spend most of the time in this environment or only after work hours. Taking into consideration a typical residence with working people, it is possible to assess comfort condition and monitor and predict values of comfort.

From the installation of different sources of sensorization through the environment it is possible to measure the impact of each individual attribute in the environment. As a personal environment those should be the best suited to assess what each user deems as comfortable. However, as users can have different meanings for comfort, there might exist some exceptions to this assertion. An environment, though not always equal it is generally categorized by a population of individuals sharing the same environment. Each environment has different requirements according to the specific tasks its users perform. An experience to demonstrate the findings of the system proposed was designed with a sensors network in place to monitor environment variables. During this experiment a presence control was instituted to add user presence as an attribute. The description of the summary of the data gathered is available in table 1.

The analysis of well-being is inherently different from home and professional con-figurations due to the fact that the context alters comfort values. Though some of the alterations remain within the acceptable range they can be used to introduce differences in the well-being analysis. 
Table 1. Summarized data retrieved from the environment sensor network

\begin{tabular}{|l|l|l|l|l|}
\hline & Mean & Standard Deviation & Max & Min \\
\hline Temperature & $19,98{ }^{\circ} \mathrm{C}$ & $1,68{ }^{\circ} \mathrm{C}$ & $24,88^{\circ} \mathrm{C}$ & $17,99{ }^{\circ} \mathrm{C}$ \\
\hline Luminosity & $122,53 \mathrm{lux}$ & $102,49 \mathrm{lux}$ & $632,00 \mathrm{lux}$ & $0,00 \mathrm{lux}$ \\
\hline Humidity & $45,69 \%$ & $7,39 \%$ & $63,19 \%$ & $38,86 \%$ \\
\hline Number of People & 2,27 & 1,70 & 10,00 & 0,00 \\
\hline
\end{tabular}

\subsection{Personal Assessment}

Well-being through environment alone is an incomplete study as it disregards the effects of user interaction and user behaviour. Under normal circumstances, well-being should generate values inside the satisfactory range for attributes being measured. Considering one individual alone, the existence of normal range values for environment and abnormal for the personal sensing might lead to the suspicion of something not right with the individual thus impairing well-being.

On table 2, a set of indicators gathered from a personal sensorization hardware reveals the normal range of attribute date for a given individual. His historic data re-veal how comfortable he is by assessing most common values after some period of time. As the indicator values go up or down the mean and standard deviation being considered it detects abnormal behavioural pattern and uses majority votes to decide whether it is really an uncomfortable behaviour or not.

A practical validation test can be made using records from environment and testing them against such data. It was perceived that environment conditions only directly affect personal attributes the most when they differ significantly under normal circumstances that is avoiding considerations about individual's state of mind. Environment variables ranging inside the comfort zone are less likely to produce chances in the normal values from personal sensorization.

Table 2. Summarized data retrieved from the user sensor network

\begin{tabular}{|l|l|l|l|l|}
\hline & Mean & $\begin{array}{l}\text { Standard } \\
\text { Deviation }\end{array}$ & Max & Min \\
\hline Steps & 4177,80 & 7537,96 & 5080,00 & 3245,00 \\
\hline Blood Pressure & $78,42 \mathrm{bpm}$ & $19,11 \mathrm{bpm}$ & $125,00 \mathrm{bpm}$ & $58,00 \mathrm{bpm}$ \\
\hline Oximetry & $97,45 \%$ & $1,02 \%$ & $100,00 \%$ & $95,00 \%$ \\
\hline PMV & $-0,79$ & 0,86 & 1,20 & $-1,72$ \\
\hline
\end{tabular}

\subsection{Well-Being Assessment}

Well-being assessment is employed in a two-phase strategy. First, the analysis of critical conditions through environment sensory data and secondly, the analysis of comfort. At the initial phase, a thorough analysis about each individual attribute is made to make sure that each of the sensorized values are nonrisk values towards human. This evaluation is made both to environment and personal attributes as shown in table 3. It is important to deal with information quality and validity. If the evaluation fails at this stage 
the uncomfortable setup is immediately generated. Even if there is only one of the attributes outside what is considered safe range, the resulting classification is deemed non satisfactory and a $0 \%$ well-being is issued as life might be endangered.

Following on to the second phase, with each user profile and environment profile, a match between activities and each attribute available is analysed. In the sample test, the temperature and luminosity of environments where chosen. Different com-fort rules are created according to the dominant activities in each environment and the ideal configuration is assessed through ranges of values from medical, thermal and ergonomic studies present in configuration databases in the system. From this point, a weighted percentage is loaded from the database to allow different comfort attributes to have more or less impact in the well-being value. The final value of well-being is the weighted majority of the satisfaction comfort for each attribute. A percentage, less than $100 \%$ denotes that at least some attribute being measured is not within comfort values for a given time. Finally, a daily assessment considers the average of well-being satisfaction between measures.

Table 3. Comfort Reference Values

\begin{tabular}{|l|l|l|}
\hline Comfort Attribute & Lower Limit & Upper Limit \\
\hline Humidity & $20 \%$ & $60 \%$ \\
\hline Temperature & $18^{\circ} \mathrm{C}$ & $25^{\circ} \mathrm{C}$ \\
\hline Luminosity & 50 lux & 107527 lux \\
\hline Temperature perception (PMV) & -1.5 & 1.5 \\
\hline Heart Rate (Woman) & 60 & 78 \\
\hline Oximetry & $90 \%$ & $100 \%$ \\
\hline
\end{tabular}

Table 4 presents the relative percentages of time the environment was considered good for well-being according to the algorithm described with the analysis of the at-tributes and their matching to each other. It is shown that environment configurations are generally portrayed as good in well-being standing but the personal attributes are lacking more often. In the case of this experiment that is due to non-activity, and sedentary lifestyle. Its implications are that, although the environments appear well configured, the activities of users are not being considered as good for well-being. Although with this setup, if the conditions verified are not directly correlated between both environment and personal attributes, the weighted average among all attributes decides the category of well-being.

Table 4. Well-Being Assessment Experiment Analysis

\begin{tabular}{|l|c|c|c|}
\cline { 2 - 4 } \multicolumn{1}{c|}{} & $\begin{array}{c}\text { Environment } \\
\text { Well-Being }\end{array}$ & $\begin{array}{c}\text { Personal } \\
\text { Well-Being }\end{array}$ & $\begin{array}{c}\text { Well-being As- } \\
\text { sessment }\end{array}$ \\
\hline Environment & $97 \%$ & $78 \%$ & $83 \%$ \\
\hline
\end{tabular}

Historical data about environment and both environment and people can be addressed making up profiles of aggregated information through the use of dashboards. These dashboards should contain valuable information for healthcare institutions and its professionals to help with patient consult for instance. More than the experience a verbal consult with a patient can produce, a virtual conversation is something that 
includes virtual data according to sensing system available. Though the system may be assessed with simulation data, it is in the real world data applied to each scenario that it usefulness can be extensively validated. For healthcare institution it is possible to navigate, through time windows and select only values of selected attributes and obtain both a measurement dashboard as well as a well-being classification. The input in the system are dependent in the number of attributes measured by the sensor network in place and the capability of its devices. The further specialized the devices and information is the further the quality of information and the validity of these analysis.

\subsection{Considerations from well-being monitoring}

The accuracy and relevance of these system is dependent on the quality of the data and information gathered. There are plenty of devices that can track a number of attributes, which in their own yield little information but when integrated and fused together often offer explanations for user behaviour which is directly linked to well-being. Avoiding the loss of data and information from manual devices can be done interlinking devices to storage units as perceived by the internet of things. A platform to store, change data into indicators and monitor their behaviour is useful not only to monitor contexts such as well-being but also other aspects such heath, productivity, stress among others.

Large scientific projects are waking in response to the availability of data through different groups of researchers. Bolder ambitions as to record everything about environments and people that technology can offer are presented in the view of large technological organizations with the increase interest in smart devices. Their view can be summarized as gathering every information about user, user behaviour and environment through the development of intelligent devices. This creates the opportunity to use such data to mine both individual users and communities of users altogether. A downside to this is a potential privacy invasion and fear that more robust machine learning models shall be used with unethical reason such as segregation. It is here that the development of a more generic knowledge, such as the definition of wellbeing mitigates theses effects. It offer a clear representation of the values according to a logic of general well-being instead of linking two attributes together without prior objective. The assessment itself is selfexplanatory with different degrees of results from ranges within user preferences to critical results with may show direct evidence on users.

\section{Automatic Notification Schemes}

The generation of automatic notifications can be used to make the users aware of their standings and to automate the environment in relation to their surroundings. The application of notification is based both on prior knowledge by experts defining expert rules for well-being. These rules are the most basic as they are static in nature. Another type could be named dynamic rules which could take into consideration past occurrences of an attribute to advise the user based.

As a two-step approach for well-being assessment, these two rule types are applied with different meanings in this research. The first is based on the expert knowledge about attributes linked to critical conditions for well-being. These warnings are generated as soon as there is the perception that an indicator is probable to activate one of such rules. This can be triggered by sensing values for indicators near the critical threshold, or, alternatively with prediction from machine learning time-sequence models. 
The second type of rules concerns comparison of present values of indicators to past values and showing an increase or decrease on their values. If we call this the indicator value variance the trend of the indicator, we can show notifications when there is a perceptible negative trend from past events, even if it is within acceptable values. This intendeds to improve user awareness and minimize potential problems in the future.

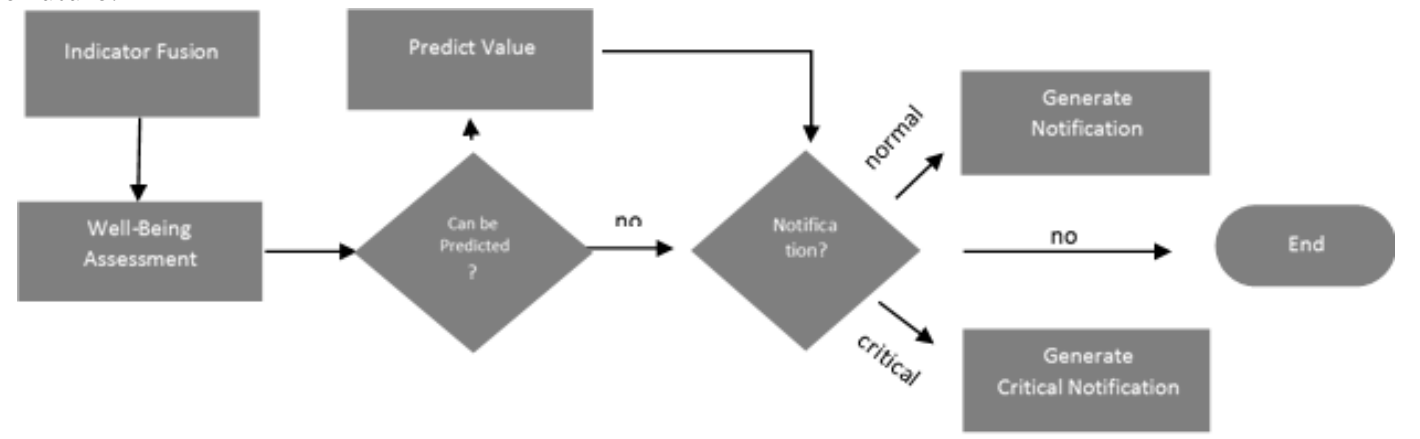

Figure 2 - Notification Generation

The flow used for notification is used as illustrated in Figure 2. The need for notification is either assessed by critical need or normal notification and is related to the well-being assessment in place. The notification itself is derived from the indicator values which violate normal well-being constraints.

The prediction of indicator values is dependent on implementation by the designer of the system. Only predictions with meaningful result shall be used. In terms of user activity one of such prediction could be number of steps predicted at the end of the day given the number of steps at mid-day for instance.

Notification itself is done directly to a communication channel either smartphone via dedicated application or simple email from the notification service.

\section{Conclusion}

The development of our well-being as human beings is something that should not be overlooked. Like other important subjects to our daily life, continuous development and improvement is desirable and an objective. Systems such as these are starting to appear through the research community and as exploratory projects within large enterprises. While not identifying themselves as health organizations, their goal is to promote the investigation of well-being through the population whilst increasing the information available. Consequently, this leads to building large databases about user behaviour and environment conditions. This also starts the study of communities and the impact of behaviours and environment on health and comfort through large sets of population strengthening existent knowledge with large validation test, but most importantly creating the opportunity to generate new and improved knowledge by analysing these records. Seemingly close behaviour in different conditions can generate different results, it can be comparable to the butterfly effect, where a small change can have great implication on the whole system towards a positive or negative result. The categorization of these conditions may improve not only wellbeing but health as well. The promises and the large research body around these themes indicates that 
these technologies and these research considerations are valid. They are even today the object of pursuit by society as a mean to improve living conditions.

A future approach to this study should include distribution analysis between the historical data on a given time window and the sensorized values. This envision may also help annotate different states of comfort through building and the people inside to better map how the environmental attributes of such buildings affect the well-being of people. As tried in the experimental framework to detect stressful moods (Silva et al., 2013), where a testbed was used to perceive different room states during the day and the classification of probable well-being.

\section{Acknowledgements}

This work has been supported by COMPETE: POCI-01-0145-FEDER-007043 and FCT - Fundação para a Ciência e Tecnologia within the Project Scope: UID/CEC/00319/2013. Additionally, it is also supported by a doctoral grant, SFRH/BD/78713/2011, also supported by FCT.

\section{References}

Atallah, L., Lo, B., \& Yang, G.-Z. (2012). Can pervasive sensing address current challenges in global healthcare? Journal of Epidemiology and Global Health, 2(1), 1-13. doi:10.1016/j.jegh.2011.11.005 http://dx.doi.org/10.1016/j.jegh.2011.11.005

Atzori, L., Iera, A., \& Morabito, G. (2010). The Internet of Things: A survey. Computer Networks, 54(15), 2787-2805. http://dx.doi.org/10.1016/j.comnet.2010.05.010

Biron, C., Ivers, H., Brun, J.-P., \& Cooper, C. L. (2006). Risk assessment of occupational stress: Extensions of the Clarke and Cooper approach. Health, Risk \& Society, 8(4), 417-429. http://dx.doi.org/10.1080/13698570601008222

Chan, M., Estève, D., Fourniols, J.-Y., Escriba, C., \& Campo, E. (2012). Smart wearable systems: current status and future challenges. Artificial Intelligence in Medicine, 56(3), 137-56. http://dx.doi.org/10.1016/j.artmed.2012.09.003

Charles, K. E. (2003). Fanger's Thermal Comfort and Draught Models Fanger's Thermal Comfort and Draught Models IRC Research Report RR-162. October, 29. doi:IRC Research Report RR-162

Choi, J., Ahmed, B., \& Gutierrez-Osuna, R. (2010). Ambulatory Stress Monitoring with Minimally-Invasive Wearable Sensors. $\begin{array}{lllll}\text { Comput. Sci. } & \text { and } & \text { Eng., } & \text { Rexas }\end{array}$ http://citeseerx.ist.psu.edu/viewdoc/download?doi=10.1.1.188.4605\&rep=rep1\&type=pdf

Fanger, P. O. (1970). Thermal comfort: Analysis and applications in environmental engineering. Danish Technical Press. Retrieved from http://books.google.pt/books?id=SOFSAAAAMAAJ

Höppe, P. (1999). The physiological equivalent temperature - a universal index for the biometeorological assessment of the thermal environment. International Journal of Biometeorology, 43(2), 71-75. Retrieved from http://www.ncbi.nlm.nih.gov/pubmed/10552310 http://dx.doi.org/10.1007/s004840050118

Krčo, S., Fernandes, J., \& Sanchez, L. (2013). SmartSantander-a smart city experimental platform. Electrotechnical Review, 3-6. Longo, M., Roscia, M., \& Lazaroiu, G. C. (2014). Innovating Multi-agent Systems Applied to Smart City, 7(20), 4296-4302. 
Parsons, K. (2010). Human thermal environments: the effects of hot, moderate, and cold environments on human health, comfort and performance. Taylor \& Francis. Retrieved from http://books.google.pt/books?id=4oxA6W_Os50C

Parsons, K. C. (2000). Environmental ergonomics: a review of principles, methods and models. Applied Ergonomics, 31(6), 581594. http://dx.doi.org/10.1016/S0003-6870(00)00044-2

Piro, G., Cianci, I., Grieco, L. a., Boggia, G., \& Camarda, P. (2014). Information centric services in Smart Cities. Journal of Systems and Software, 88, 169-188. http://dx.doi.org/10.1016/j.jss.2013.10.029

Rana, R., Kusy, B., Jurdak, R., Wall, J., \& Hu, W. (2013). Feasibility analysis of using humidex as an indoor thermal comfort predictor. Energy and Buildings, 64, 17-25. doi:10.1016/j.enbuild.2013.04.019

Silva, F., Analide, C., Rosa, L., Felgueiras, G., \& Pimenta, C. (2013). Social Networks Gamification for Sustainability Recommendation Systems. In Distributed Computing and Artificial Intelligence (pp. 307-315). Springer.

Silva, F., Olivares, T., Royo, F., Vergara, M. A., \& Analide, C. (2013). Experimental Study of the Stress Level at the Workplace Using an Smart Testbed of Wireless Sensor Networks and Ambient Intelligence Techniques. In J. Ferrández Vicente, J. Álvarez Sánchez, F. de la Paz López, \& F. J. Toledo Moreo (Eds.), Natural and Artificial Computation in Engineering and Medical Applications SE - 21 (Vol. 7931, pp. 200-209). Springer Berlin Heidelberg. doi:10.1007/978-3-642-38622-0_21

Solanas, A., Patsakis, C., Conti, M., Vlachos, I., Ramos, V., Falcone, F., .. Martinez-Balleste, A. (2014). Smart health: A contextaware health paradigm within smart cities. IEEE Communications Magazine, 52(8), 74-81. http://dx.doi.org/10.1109/MCOM.2014.6871673

Steele, R., \& Clarke, A. (2013). The Internet of Things and Next-generation Public Health Information Systems. Communications and Network, 05(03), 4-9. http://dx.doi.org/10.4236/cn.2013.53B1002

Tauseef, M. (2012). Human Emotion Recognition Using Smart Sensors. Massey University. 
